\title{
Actions for signature change *
}

\author{
Franz Embacher \\ Institut für Theoretische Physik \\ Universität Wien \\ Boltzmanngasse 5 \\ A-1090 Wien \\ E-mail: fe@pap.univie.ac.at \\ UWThPh-1995-1 \\ gr-qc/9501004
}

\begin{abstract}
This is a contribution on the controversy about junction conditions for classical signature change. The central issue in this debate is whether the extrinsic curvature on slices near the hypersurface of signature change has to be continuous (weak signature change) or to vanish (strong signature change). Led by a Lagrangian point of view, we write down eight candidate action functionals $S_{1}, \ldots S_{8}$ as possible generalizations of general relativity and investigate to what extent each of these defines a sensible variational problem, and which junction condition is implied. Four of the actions involve an integration over the total manifold. A particular subtlety arises from the precise definition of the Einstein-Hilbert Lagrangian density $|g|^{1 / 2} R[g]$. The other four actions are constructed as sums of integrals over singe-signature domains. The result is that both types of junction conditions occur in different models, i.e. are based on different first principles, none of which can be claimed to represent
\end{abstract}

*Work supported by the Austrian Academy of Sciences in the framework of the "Austrian Programme for Advanced Research and Technology". 
the "correct" one, unless physical predictions are taken into account. From a point of view of naturality dictated by the variational formalism, weak signature change is slightly favoured over strong one, because it requires less à priori restrictions for the class of off-shell metrics. In addition, a proposal for the use of the Lagrangian framework in cosmology is made.

PACS-numbers: 04.20.Cv, 04.50.+h, 04.90.+e 


\section{Introduction}

The Euclidean path integral approach to quantum cosmology [1]-[2] has led to the study of metrics that change their signature type (from Euclidean to Lorentzian) at a hypersurface $\Sigma$ (which is spacelike with respect to the Lorentzian side). Originally, metrics emerging in a WKB-approximation of quantum gravity as "real tunneling geometries" were studied [3]-[5]. The junction condition in this case is that the extrinsic curvature at $\Sigma$ vanishes, when computed within the embedding from either side (or appropriate generalizations thereof when matter fields are added, typically $\partial_{t} \phi=0$ at $\Sigma$ with $\partial_{t}$ the affine comovig parameter derivative). We will refer to this type of junction condition as strong signature change.

Later, is was proposed that a classical change of signature type is possible within a more relaxed framework as well, with junction condition requiring the extrinsic curvature on slices near $\Sigma$ to be continuous [6]-[7], hence possibly non-zero, as opposed to the older approach (the corresponding generalization to additional matter fields being typically $\partial_{t} \phi=$ continuous). We denote this situation as weak signature change. For some work which has been done within this approach, see e.g. Refs. [8]- 17].

Since then, many aspects of these two types of junction conditions have been studied (see for example, in addition to the works quoted above, Refs. [18]-[24]), and occasionally the question which of these is the "correct" one is adressed (more or less explicitly). Regrettably, the different points of view are sometimes not very clearly based on different underlying assumptions. This has led to mutual accusation of "errors" among researchers to an extent that seems to rather supply misunderstandings than to clarify matters. The present piece of work is devoted to the study of different assumptions (leading to different junction conditions) from a Lagrangian point of view, hence to the clarification of the question which first principles lead to which type of junction condition.

One plausible point of view (taken e.g. by Hayward [4, 23]) is to consider Einstein's field equations ( $R_{\mu \nu}=0$ in a distributional sense) as the fundamentals (first principles) of a model of classical signature change. (We will only consider the vaccum case here - except for Section 9, where we add a cosmological constant -, as the inclusion of additional matter fields will not at all affect the principal arguments and results given). To be more precise: by $R_{\mu \nu}$ we mean the standard Ricci tensor as computed using the standard formulae as e.g. given in Ref. 25] (whose conventions we are using) and inserting a metric that displays a change of signature type on 
a hypersurface $\Sigma$. It has been shown in Refs. [4], [18] that the resulting Einstein equations may be interpreted in terms of generalized functions (distributions) and yield the junctions conditions of strong signature change as their $\delta$-function part (see also equations (2.7)-(2.9) below). We will call these the "full" Einstein equations in order to avoid confusion with the regular, single-signature (Lorentzian or Euclidean) Einstein equations that have to be satisfied off $\Sigma$ anyway.

However, there is another, perfectly legitimate point of view, namely the Lagrangian one. May classical signature change be described within a proper variational framework, i.e. is there a generally covariant action functional $S$ such that $\delta S=0$ has signature type changing metrics as solutions? In some sense, this point of view is even more fundamental that the field equations approach, because it gives quantization as well as coupling to realistic field therories a conceptually firm basis. The former is of course only important if quantisation is a goal. This seems to be one source of misunderstandings: Shall we quantize "classical signature change", as was envisaged in Ref. [14], and expect oscillating WKB-solutions even in the Euclidean domain, or shall we consider "classical signature change" as a classical model of quantum tunneling in quantum cosmology (that needs not be quantized at all)? Some authors (see e.g. Ref. [12]) explicitly emphasize that their intention is not a classical version of quantum cosmology, and these statements seem to have been overlooked by others. Here, we are just interested in possible generalizations of general relativity that admit a change of signature, hence we do not favour one of the two types of junction conditions, and we do not pre-assume any relation of such a model to quantum cosmology (except for some speculations in Section 9).

Let us note as a starting point that we have several assumptions for first principles at our disposal (based on Einstein's equations and based on action principles), and that we do not know (so far) which of them is the "correct" one. We are just interested in the consequences of each choice.

Although sometimes action functionals are written down in the recent literature on signature change (see e.g. Refs. [4], [9]), their variations and hence their implications have, to my knowledge, not been worked out properly so far. Maybe, some particular features that arise here have simply been overlooked. One such feature is, as we will see in Section 2, the possibility that

$$
\delta S_{E H}=\int_{\mathcal{M}} d^{n} x|g|^{1 / 2} G_{\mu \nu}[g] \delta g^{\mu \nu}+\text { additional terms },
$$

where $S_{E H}$ is the standard Einstein-Hilbert action with a signature changing metric inserted (although this provides some subtleties to which we will return later on), and 
the additional terms contain generalized functions with support on $\Sigma$. Thereby, the variations vanish outside some domain $\mathcal{N}$, but not necessarily on $\Sigma$. (The variational prescription actually requires $\mathcal{N}$ to be essentially arbitrary. The appearance of the additional terms in (1.1) may of course be circumvented by choosing $\mathcal{N}$ to have empty intersection with $\Sigma$, but this would not lead to a Lagrangian derivation of signature change).

From standard (Lorentzian signature) general relativity one is used to the field equations $G_{\mu \nu}=0$ (or $R_{\mu \nu}=0$ ) being equivalent to the (variational) principle that the Einstein-Hilbert action is stationary, $\delta S_{E H}=0$. However, if the metric is allowed to change its signature type, this is not necessarily the case, as (1.1) shows. As we will see in Section 2, the reason for this fact is a possible "mismatch" between the integrand (i.e. the Lagrangian density) and the volume element. However, the particular form of the "additional terms" in (1.1) depends on the class of admissible metric variations as well as on the precise way the product between the Ricci scalar and the volume element is defined, and there is one way of doing it such that they vanish. In this way, we will arrive at two possible definitions of the Einstein-Hilbert action for signature changing metrics. Moreover, in standard general relativity, one may subtract a boundary term from the Einstein-Hilbert action (it is sometimes called the Gibbons-Hawking boundary term; see Ref. [26] for the appearance of this modified action in quantum gravity). In the case of signature change, such a modification ensures all integrations to be performed over regular functions only, but changes the class of admissible metric variations and seems to have greater impact on the character of the model than in standard general relativity.

This amounts to re-examine the perspectives for the use of variational principles when a change of signature is envisaged. Let us provide some arguments why $S_{E H}$ (which is, as mentioned, not even a unique concept) is not the only possible action functional.

The main thing we can be sure about is that for the case of a purely Lorentzian metric, the action functional shall coincide with one of the standard actions for general relativity $\left(S_{E H}\right.$ or $S_{E H}$ with the Gibbons-Hawking boundary term removed). Furthermore, the action functional for the purely Euclidean case shall be of the same type (the Euclidean Einstein-Hilbert action). However, here we have the choice of an additional sign in front of it. There is no physical à priori reason that would forbid us to assume the Euclidean part of the action to appear with a minus sign relative to the Lorentzian part. A formal justification of such an assumption is that the metric signature is a coordinate-independent (hence "covariant") concept and 
thus might in principle provide a constituent of an action integral.

A further issue where the possibility of a choice appears is the question how to treat the integration across the signature change hypersurface $\Sigma$. In view of the distributional character of the full Ricci scalar at $\Sigma$, one might split the action functional into two contributions, each being defined as an integral over the respective single-signature domain. The advantage of this approach is that the integrals appearing are perfectly well-defined, and no distibutions have to be dealt with. There is nothing exotic with such a concept. On the contrary, it applies to standard (Lorentzian signature) general relativity as well: Assume that any hypersurface cuts the total space-time manifold into two pieces, and postulate a variational principle defined by the sum of the respective Einstein-Hilbert actions of the two pieces. Then, under mild continuity and differentiability conditions for the total class of (off-shell) metrics, one recovers Einstein's field equations. Let us call this the "additivity property".

Here a comment on our use of the words "on-shell" and "off-shell" (that originally referred to the mass shell condition for particles in special relativity) is in place: By "off-shell", we denote those metrics which are considered from the outset, before field equations or variational principles are imposed. The variations are performed within this class of metrics. The physical solutions (i.e. the solutions of field equations or variational principles) are called "on-shell". The precise definition of the class of offshell metrics depends on the model considered (this definition is actually a part of the model, along with the formal action integral) and is usually made such that the action $S[g]$ is well-defined and the variational principle $\delta S[g]=0$ admits nontrivial solutions. However, there is always a certain freedom to modify models by redefining the class of off-shell metrics.

Thus, combining these possibilities for action functionals, we arrive at eight candidates. If $\epsilon$ denotes the metric signature $(\epsilon=1$ for a Lorentzian metric and $\epsilon=-1$ for a Euclidean one), these eight candidates are constructed as follows: $S_{1} \equiv S_{E H}$ is obtained by integration over the total Ricci scalar $R[g], S_{2}$ by integration over $\epsilon[g] R[g]$. Thereby, the absolute value of the metric determinant $|g|^{1 / 2}$ is defined as a (continuous) object and thereafter multiplied by $R[g]$ and $\epsilon[g] R[g]$, respectively. $S_{3}$ and $S_{4}$ are anologous constructions, but with the product $|g|^{1 / 2} \times R[g]$ defined in a different way. We denote all these four candidates as "singular actions", because the integrands have to be interpreted in terms of generalized functions (if possible). $S_{5}$ is the integral over $R[g]$, but broken into two regular contributions from the Lorentzian and the Euclidean domain, and $S_{6}$ is the corresponding expression using 
$\epsilon[g] R[g]$. Finally $S_{7}$ and $S_{8}$ are the regular two-domain constructions based on $R[g]$ and $\epsilon[g] R[g]$, but with appropriate boundary terms subtracted. The last four candidates will be called "regular actions".

The division into singular and regular actions will prove important as the former are plagued by ill-defined nonlinear combinations of distributions. Our attempts to overcome these problems are somewhat heuristic and unsatisfactory, and in all cases they are successfull, we will end up effectively within the framework of regular actions. This has of course some impact on our notion of "naturality", and among our results maybe the most appealing one will be a relation between $S_{8}$ and the "additivity property".

So far, all eight candidate actions have been introduced formally (except for the subtleties distinguishing $S_{1}$ and $S_{2}$ from $S_{3}$ and $S_{4}$ ). It is clear that off the hypersurface $\Sigma$ of signature change, they just procuce the single-signature (Lorentzian or Euclidean) Einstein equations. The crucial point is what they predict at (more precisely: across) $\Sigma$. In order to examine their ability to describe signature change within a reasonable variational framework, we have to adress several questions to each of them: (i) In which sense is it well-defined? (ii) What is the class of off-shell metrics, i.e. the set of metrics within which the variations are performed? Since all variations are infinitesimal, this question may in a sloppy way be rephrased as: What is the class of off-shell variations? (By "off-shell", we mean "not necessarily a solution", whereas "on-shell" denotes the solutions of the variational principle). (iii) Which type of junction condition follows? These questions will be answered for all eight candidate actions.

Our results may be summarized as follows: The answers to the questions posed above will essentially be that the actions based on $R[g]\left(S_{1}, S_{3}, S_{5}\right.$ and $\left.S_{7}\right)$ are associated with strong, and those based on $\epsilon[g] R[g]\left(S_{2}, S_{4} S_{6}\right.$ and $\left.S_{8}\right)$ are associated with weak signature change, although on a very different level of well-posedness and naturalness within the usual spirit of Lagrangian formulations. The main problems arise from the fact that for some cases $\delta S=0$ admits nontrivial solutions only if severe restrictions on the class of off-shell metrics are imposed. Disappointingly, $S_{1} \equiv S_{E H}$ (in the interpretation that $|g|^{1 / 2}$ is a well-defined object by its own before multiplied by $R[g]$ ) defines a proper variational problem only if the strong junction conditions are imposed from the outset on the class of off-shell metrics (which is clearly an unpleasant feature within a variational formulation). A similar problem is encountered with $S_{2}, S_{4}$ and $S_{5}$ (although in the two former cases an ad hoc but not very beautiful regularization scheme might save the situation). Altogether, these 
results seem to indicate that the singular actions $S_{1}, S_{2}, S_{4}$ and $S_{5}$ are ruled out. The singular action $S_{3}$ (which turns out to be essentially the same as $S_{7}$ ) defines a model for strong signature change, once a differentiability condition is imposed on the class of off-shell metrics. (Such a condition is acceptable although not very appealing). This model then leads directly to the full (distributional) Einstein equations. Its peculiar feature is that the Lagrangian density is not constructed as an ordinary product $|g|^{1 / 2} \times R[g]$. (To what extent this is a "disadvantage" is a matter of philosophy). The candidates $S_{6}$ and $S_{7}\left(\equiv S_{3}\right)$ define sensible models only if the offshell metrics satisfy a differentiability condition, and hence give rise to more or less acceptable models for weak and strong signature change, respectively. The winner is $S_{8}$, which only needs a continuity condition for the off-shell metrics and leads to weak signature change. This last action is a natural generalization of what one uses in Euclidean path integral quantum cosmology [1], [2], i.e. with the usual GibbonsHawking boundary term [26] subtracted, and with an additional minus sign for the Euclidean sector. All these results are valid in an arbitrary number of space-"time" dimensions $n \geq 3$.

In terms of a (very simplifying) toy model, the actions based on $R[g]$ correspond to the Lagrangian (let $\epsilon \equiv \operatorname{sgn}(x)$ )

$$
\mathcal{L}_{1}^{\text {toy }}=\frac{1}{2} \epsilon \dot{x}^{2}-U(x),
$$

whereas the actions based on $\epsilon[g] R[g]$ correspond to the Lagrangian

$$
\mathcal{L}_{2}^{\text {toy }} \equiv \epsilon \mathcal{L}_{1}^{\text {toy }}=\frac{1}{2} \dot{x}^{2}-\epsilon U(x)
$$

The junction conditions are (if the sign changes at $t=0) \dot{x}=0$ (strong) and $\dot{x}(-0)=\dot{x}(+0)($ weak $)$, respectively.

As a by-product of the pattern of signs emerging, we will see that the regular candidate actions $S_{6}$ and $S_{8}$ (both associated with weak signature change) may be formulated for the single-signature case as well and precisely provide the statement that the single-signature Einstein-Hilbert action may be broken into a sum of integrals and still produces Einstein's field equations (as was mentioned above as the "additivity property"). Thus, from the point of view of the sign structure, the invariant $\epsilon[g] R[g]$ seems to provide a variational principle in a way as natural as $R[g]$. 
This may be illustrated in terms of the toy model given above. The natural action to consider is (let $\epsilon_{ \pm}(t)$ be $\operatorname{sgn}(x(t))$ for $t>0$ and $t<0$, respectively)

$$
S^{\text {toy }}=\epsilon_{-} \int_{t_{\min }}^{0} d t \mathcal{L}_{1}^{\text {toy }}+\epsilon_{+} \int_{0}^{t_{\max }} d t \mathcal{L}_{1}^{\text {toy }}
$$

Then the sign combinations with $\epsilon_{+}=-\epsilon_{-}$produce the analogue of weak signature change, whereas the sign combinations with $\epsilon_{+}=\epsilon_{-}$just illustrate the "additivity property" (weak signature change thus appearing as a generalization thereof). We should add that this toy model displays only part of the structure encountered in gravity (namely as far as the lapse and shift degrees of freedom, a divergence term and some further subtleties are ignored).

In this way one might, contrary to the most approaches to signature change, get the intuitive feeling that the Ricci scalar $R[g]$ as it is used in the Einstein-Hilbert action for standard general relativity is in fact $\epsilon[g] R[g]$, but with $\epsilon[g]=1$ inserted, since the metric is Lorentzian "today". Hence, there is no reason to consider those actions which display a non-standard sign in the Euclidean domain as less natural. This concludes the summary of the results obtained.

There may be, however, yet other possibilities for action functionals that we have not considered here (as e.g. the inclusion of further boundary integrals that would act like a gauge-fixing term, enforcing the strong junction conditions). Moreover, we have not included matter fields (except for a cosmological constant in Section 9) because their presence would neither change our arguments nor the structure of the results. In particular the generalization to bosonic matter fields seems to be staightforward.

Let us add the conceptual remark that in a proper Lagrangian framework the hypersurface $\Sigma$ has the status of a variable too, and the metric variations $\delta g_{\mu \nu}$ have to be supplemented by variations of the location of the hypersurface, symbolically denoted as $\delta \Sigma$. However, it will turn out in Section 8 that for the most reasonable candidates, $S_{7}$ and $S_{8}$, this aspect does not produce any new information, so that in the main part of this paper $\Sigma$ is held fixed (and described by the equation $\left.x^{0} \equiv t=0\right)$. As a consequence of $\Sigma$ being part of the dynamical variables, the Lagrangian formulation does not tell us whether a classical change of signature actually will occur. Consider e.g. a Lorentzian metric that develops all necessary conditions for a signature change at some spacelike hypersurface $\Sigma$. Then two solutions are possible: one with and one without signature change. This is a true 
non-uniqueness of solutions to the Lagangian formulation, and it is already present in an approach based on field equations and junction conditions. Presumably only the quantization or an ad hoc assumption can remove it. In Section 9, we will give an example for such an assumption (it essentially states - in a cosmological framework - that signature change will always occur whenever possible, and thus render the metric Euclidean/Lorentzian in the sectors of superspace that are usually referred to as Euclidean/Lorentzian). This type of non-uniqueness is not an obstruction, and a comparable thing happens in classical string theory [27], where the worldsheet topology between a given initial and final configuration is not unique, due to "unpredictable" splitting and joining effects of the pieces of the string.

In order to avoid possible confusion with another "classification" of methods, we should make a further, more technical remark. There have been developed two notions of classical signature change, labelled as the "continuous" and the "discontinuous" approach (see e.g. Refs. [6], 18]). However, the difference between these approaches is to some extent only a coordinate transformation [18, [22]. The prototype situation is given by the two forms

$$
-\operatorname{sgn}(t) d t^{2}=-\tau d \tau^{2}
$$

for the pure "time"-part of the metric, with $t$ being the affine comoving parameter and $\tau$ the corresponding "continuous approach" coordinate. Hence, $\operatorname{sgn}(t)=\operatorname{sgn}(\tau)$ and $|t|=(2 / 3)|\tau|^{3 / 2}$. The distinction between them will not be important at all. This comes from the fact that only the limits of quantities (like the metric) as computed within the embedding from either side are relevant for the Lagrangian point of view (together with the distributions that are generated by differentiating discontinuous functions with respect to $t$ ), but we never need to specify, say, the particular value of $g_{00}$ at $\Sigma$ (i.e. at $t=0$ ), even if $g_{00}$ is discontinuous. As a consequence, a simple coordinate transformation will translate any statement made in the "discontinuous" approach into one made in the "continuous" approach, and vice versa. We prefer to use the language of the "discontinuous" approach, because Gaussian coordinates $\left(g_{00}= \pm 1, g_{0 i}=0\right)$ are particularly easy to handle. Note however that the two coordinates $t$ and $\tau$ are related to two different notions of "differentiability". The existence of $\partial_{t} f$ is not equivalent to the existence of $\partial_{\tau} f$ at $t=\tau=0$, for any function $f$. In the Lagrangian framework we will not presuppose any such condition for the physical variables (in particular the spatial metric), and the natural notion of differentiability associated with this approach will be the one with respect to $t$. From the Lagrangian point of view there is nothing special about 
$\tau$, whereas $t$ has a preferrred status as the local orthogonal metric distance from $\Sigma$.

The paper is organized as follows: In Section 2, we give some preliminaries, in particular the variations of some Lagrangian density expressions, when signature change is allowed. In Section 3, the definition of the list of candidate actions is given. Section 4 is devoted to the study of the singular actions $S_{1}$ and $S_{2}$, Section 5 is concerned with the singular actions $S_{3}$ and $S_{4}$. The regular actions $S_{5}$ and $S_{6}$ (using the total single-signature Ricci scalars) are considered in Section 6, the regular actions $S_{7}$ and $S_{8}$ (being based on the subtraction of a surface integral) are dealt with in Section 7. In Section 8, we include the variation of the hypersurface $\Sigma$. Section 9 deals with a coordinate dependent but instructive form of $S_{7}$ and $S_{8}$ and a proposal for a resolution of the non-uniqueness problem within the frawework of cosmology. Finally, Section 10 contains some concluding remarks.

\section{Variations}

In order to define a convenient $(n-1)+1$ split formalism with coordinates $x^{\mu} \equiv$ $\left(t, x^{i}\right) \equiv(t, \mathbf{x})$, assume the choice of a slicing $\Sigma_{t}$ of hypersurfaces defined by constant values of $t$, such that a signature change occurs at $\Sigma \equiv \Sigma_{0}$. (One may in fact consider several signature changes, in which case it is reasonable to parametrize their hypersurfaces as $\Sigma_{j} \equiv \Sigma_{t_{j}}$ ). Assume the hypersurface(s) of signature change to be spacelike with respect to the Lorentzian side(s). Furthermore, let $n^{\mu}$ be the (unique) vector field orthogonal to the slices, "future" directed with respect to $t\left(n^{0} \geq 0\right)$ and having the square $n^{\mu} n_{\mu}=-\epsilon$. Later on, we shall choose $\epsilon=1$ for the Lorentzian and $\epsilon=-1$ for the Euclidean domains. However, in view of the distributional character of some quantities appearing, we treat $\epsilon$ as an arbitrary function of the "time" coordinate, $\epsilon \equiv \epsilon(t)$, in all general formulae derived here. Then $n_{\mu}=-\epsilon N \partial_{\mu} t$ (or $\left.n^{0} \equiv n^{\mu} \partial_{\mu} t=1 / N\right)$ defines the lapse function $N \geq 0$. The induced metric (first fundamental form) on $\Sigma_{t}$ is given by $h_{\mu \nu}=g_{\mu \nu}+n_{\mu} n_{\nu} / \epsilon$, the extrinsic curvature (second fundamental form) by $K_{\mu \nu}=-h_{\mu}^{\rho} h_{\nu}^{\sigma} \nabla_{\rho} n_{\sigma}$. This is actually a sloppy notation, and in order to be more precise we mention that the "true" extrinsic curvature is $K_{\mu \nu} /|\epsilon|^{1 / 2}$. It is a "covariant" object, and it proves useful if one tries to reproduce the results of this paper in a manifestly "continuous" approach (which amounts to set $\epsilon(t)=t$ ). We will rather use a manifestly "discontinuous" language in which $\epsilon$ (after a sequence of steps during which it is treated as arbitrary function) is set 
equal to a step function (preferably \pm 1 ), and $N$ is continuous.

The spatial components of $n^{\mu}=\left(1 / N,-N^{i} / N\right)$ (or the components $h_{0 i}=N_{i}$ ) define the shift vector. The metric reads

$$
\begin{aligned}
d s^{2} & =-\epsilon N^{2} d t^{2}+h_{i j}\left(d x^{i}+N^{i} d t\right)\left(d x^{j}+N^{j} d t\right) \\
& =\left(-\epsilon N^{2}+N^{i} N_{i}\right) d t^{2}+2 N_{i} d t d x^{i}+h_{i j} d x^{i} d x^{j},
\end{aligned}
$$

where the induced metric $h_{i j}$ appears as an $(n-1)$-object and serves (togeher with its inverse $h^{i j}$ ) to raise and lower spatial indices (e.g. $N_{i}=h_{i j} N^{j}$ ). The components $N, N_{i}$ and $h_{i j}$ are functions of all coordinates. Note that discontinuous $\epsilon$ (although producing $\delta$-distributions wherever $\dot{\epsilon}$ appears) does not necessarily imply discontinuous metric components (as the example $\epsilon(t)=\operatorname{sgn}(t), N(t)=|t|^{1 / 2}$ shows: this is equivalent to $\epsilon(t)=t, N(t)=1$ and thus another way to implement the "continuous" approach). The spatial version of the extrinsic curvature is

$$
K_{i j}=\frac{1}{2 N}\left(N_{i \mid j}+N_{j \mid i}-\partial_{t} h_{i j}\right)
$$

where denotes the covariant derivative with respect to $h_{i j}$. Furthermore, we define $K=K_{i}^{i} \equiv h^{i j} K_{i j}$. Note that $\epsilon$ has not been constrained so far (apart from $\partial_{i} \epsilon=0$ ). Only if $\epsilon= \pm 1$, the above construction coincides with the standard $(n-1)+1$ split formulation (see e.g. Refs. [25], 28] for the Lorentzian signature case).

The determinant of (2.1) is $g \equiv \operatorname{det}\left(g_{\mu \nu}\right)=-\epsilon N^{2} h$, with $h \equiv \operatorname{det}\left(h_{i j}\right)$. The volume element for the integration in the action shall be defined as $|g|^{1 / 2} d^{n} x$. Putting $\epsilon= \pm 1$ in the respective domains amounts to set $|\epsilon|=1$ and hence $|g|^{1 / 2}=N h^{1 / 2}$. This definition aims at the construction of the volume element as a well-defined quantity by its own. (This construction of a volume element is quite natural and is occasionally written down explicitly for signature changing metrics, see e.g. Ref. [13]). Its prefactor is continuous (as long as $N$ and $h^{1 / 2}$ are), and an integration is defined by multiplying this object by the integrand (which may be a distribution and is considered as a well-defined object by its own as well). The Einstein-Hilbert action is such an integral, the integrand being the Ricci scalar $R[g]$ (a combination of ordinary functions and $\delta$-distribution; see equation (2.3) below). This leads to the version $S_{1}$ of the Einstein-Hilbert action.

However, there is an other possibility that will lead to a different version of the Einstein-Hilbert action (namely to $S_{3}$ ). It consists of considering the "product" 
$|g|^{1 / 2} R[g]$ as the result of a limiting process, in which first $\epsilon$ is treated formally as if it were an arbitrary function, and in a second step the resulting formal expression for $|g|^{1 / 2} R[g]$ is given some sense. This in turn amounts to define the volume element formally as $|\epsilon|^{1 / 2} N h^{1 / 2} d^{n} x$. When varying the Einstein-Hilbert action defined in this way, derivatives $\partial_{t}|\epsilon|$ appear. Although this is zero for a step function in the usual sense of distributions, a complication arising is that it has to be multiplied by further $\epsilon$-terms, so that the final expression is not well-defined as it stands and has to be interpreted. One interpretation is to insert $|\epsilon|=1$, which renders $\partial_{t}|\epsilon|=0$ (hence reproduces the choice $|g|^{1 / 2}=N h^{1 / 2}$ ), but another interpretation is to identify $\left(\partial_{t}|\epsilon|\right) /|\epsilon|$ with $\left(\partial_{t} \epsilon\right) / \epsilon$, and this will provide a different action. Hence, we keep in mind that we have two (different) definitions for volume integrals at hand. In both schemes it is important not to use identities like $1 / \epsilon=\epsilon$ or $\epsilon^{2}=|\epsilon|=1$ too early. As an example, $\partial_{t}(1 / \epsilon)$ is to be evaluated as $-\dot{\epsilon} / \epsilon^{2}$. The reason for these subtle issues is that distributions occur in a non-linear manner. We decide to keep such expressions as they stand (in particular distinguishing between $\epsilon$ and $1 / \epsilon$ ) in all formulae throughout the whole paper, unless obvious or stated otherwise. Occasionally, one has the freedom to interpret ill-defined objects in different ways: the volume element problem is an example of such a case. (Rephrased in the quantum field theory jargon this means that one may apply different "regularization" schemes). All these subtleties (e.g. in defining the behaviour of the Lagrangian density across $\Sigma$ ) are of course irrelevant for the regular actions $S_{5}, \ldots S_{8}$, who avoid any problems of this sort from the outset.

Locally, one can always choose coordinates such that $N=1$ and $N_{i}=0$ (thus $x^{0} \equiv t$ being the local orthogonal metric distance from $\Sigma$, or - in other words the "affine comoving parameter"). Such coordinates are referred to as Gaussian ones, and (once $\Sigma \equiv \Sigma_{0}$ is given) they are unique up to $t$-independent spatial transformations $x_{\text {new }}^{i}=X^{i}(\mathbf{x})$. In a Gaussian coordinate system, we require $h_{i j}$ to be strictly positive, hence $h>0$.

The Ricci scalar with respect to (2.1) is given by (cf. Refs. [25], 28])

$$
\begin{aligned}
R[g]= & R[h]+\frac{1}{\epsilon}\left(K^{i j} K_{i j}+K^{2}\right)-\frac{2}{N} N^{\mid i}{ }_{\mid i} \\
& -\frac{\dot{\epsilon}}{\epsilon^{2} N} K+\frac{1}{N}\left(\partial_{t}-N^{i} \partial_{i}\right)\left(-\frac{2}{\epsilon} K\right),
\end{aligned}
$$

where again $\epsilon \equiv \epsilon(t)$ has been treated as an arbitrary function. If $\epsilon= \pm 1$ is a step function, one could insert $\epsilon^{2}=1$ and $\dot{\epsilon}$ a $\delta$-function. (Let us repeat that some 
caution is necessary here: one is not allowed to insert the same step function for $1 / \epsilon$ in the last expression before the "time"-derivative is carried out). In a coordinate system with zero shift and $N \equiv N(t)$, our expression for $R[g]$ coincides with the one given by Kossowski and Kriele (in the appendix of Ref. [18]). Also, these authors adopt - in part of their paper - the "discontinuous" language and proceed along similar lines when computing the energy-momentum tensor. Clearly, $R[g]$ is only a well-defined distribution if $K$ (which appears multiplied by $\dot{\epsilon} / \epsilon^{2}$ ) is continuous. This implies a differentiability condition for the class of off-shell metrics for all models using the full Ricci scalar and an integration over the total manifold. We will impose such restrictions in detail later on, when discussing the particular models. However, we note that just this type of ill-definedness of quantities for a large class of metrics provides the major drawback for all singular actions.

In order to proceed, we multiply $R[g]$ by $N h^{1 / 2}$ (which is $|g|^{1 / 2}$ in the interpretation that $|\epsilon|=1$ is to be inserted). Thus, the integrand for the action $S_{1}$ (and a basic quantity from which the other actions are constructed as well)

$$
\mathcal{L}=N h^{1 / 2} R[g]
$$

is given by

$$
\begin{aligned}
\mathcal{L}= & N h^{1 / 2} R[h]+\frac{1}{\epsilon} N h^{1 / 2}\left(K^{i j} K_{i j}-K^{2}\right)-\frac{\dot{\epsilon}}{\epsilon^{2}} h^{1 / 2} K \\
& +\partial_{t}\left(-\frac{2}{\epsilon} h^{1 / 2} K\right)+\partial_{i}\left(\frac{2}{\epsilon} h^{1 / 2} N^{i} K-2 h^{1 / 2} N^{\mid i}\right) .
\end{aligned}
$$

In all integrations over the spatial coordinates, divergence terms like the last contribution in (2.5) will be omitted. The role of momenta in the canonical formulation is played by the spatial tensor density

$$
\widetilde{\pi}^{i j}=\frac{\partial}{\partial \dot{h}_{i j}} N h^{1 / 2}\left(K^{k l} K_{k l}-K^{2}\right)=h^{1 / 2}\left(K h^{i j}-K^{i j}\right)
$$

or its negative (according to the sign in front of $\mathcal{L}$ and the sign of $\epsilon$ ).

In order to take a look at Einstein's field equations $R_{\mu \nu}=0$, we write down the components of the Ricci tensor (for simplicity in a coordinate system in which $\left.N_{i}=0\right)$

$$
R_{00}[g]=N \partial_{t} K-\frac{\dot{\epsilon}}{2 \epsilon} N K-N^{2} K^{i j} K_{i j}+\epsilon N N_{\mid i}^{\mid i}
$$




$$
\begin{aligned}
R_{0 i}[g]= & N\left(\partial_{i} K-K_{i \mid j}^{j}\right), \\
R_{i j}[g]= & R_{i j}[h]+\frac{\dot{\epsilon}}{2 \epsilon^{2} N} K_{i j} \\
& -\frac{1}{\epsilon}\left(\frac{1}{N} \partial_{t} K_{i j}+2 K_{i}^{l} K_{l j}-K K_{i j}\right)-\frac{1}{N} N_{\mid i j} .
\end{aligned}
$$

Before going on to the Lagrangian approach, we observe that Einstein's equations decouple into a regular part (the single-signature field equations) and a contribution proportional to $\dot{\epsilon}$ (which is a $\delta$-distribution at $\Sigma$, where $\epsilon$ is discontinuous). In Gaussian coordinates, the latter reduces to $\partial_{t} h_{i j}=0$ at $\Sigma$, hence the junction conditions for strong signature change. This fact provides the basis from which the concept of weak signature change is usually critizised as not satisfying "the correct" field equations (see e.g. Ref. [23]). Such criticism is of course obsolet, once "the" field equations are defined in terms of variational problems, both strong and weak signature change being possible and associated with different actions.

The Lagrangian formulation amounts to compute variations of the action due to infinitesimal changes off a given metric. We will assume this given metric to be expressed in terms of Gaussian coordinates. However, the variations off this metric must be generic, i.e. they induce nontrivial lapse and shift functions, although at an infinitesimal level. Hence, we must use the full expression (2.5) when varying $\mathcal{L}$. As independent variations we choose $\delta N, \delta N_{i}$ and $\delta h_{i j}$, and we use

$$
A^{i j}=N^{i \mid j}-\frac{N^{\mid i}}{N} N^{j}
$$

as an abbreviation. For the sake of generality, we display the full structure of $\delta \mathcal{L}$ :

Variation with respect to the lapse:

$$
\delta \mathcal{L}=\mathcal{E} \delta N+\frac{\dot{\epsilon}}{\epsilon^{2} N} h^{1 / 2} K \delta N+\partial_{t}\left(\frac{2}{\epsilon N} h^{1 / 2} K \delta N\right)+\partial_{i} \mathcal{U}^{i} .
$$

Variation with respect to the shift: The independent variation is $\delta N_{i} \equiv \psi_{i}$, and we set $\psi^{i} \equiv h^{i j} \psi_{j}$.

$$
\begin{aligned}
\delta \mathcal{L}= & \mathcal{E}^{i} \psi_{i}-\frac{\dot{\epsilon}}{\epsilon^{2} N^{2}} h^{1 / 2} N^{\mid i} \psi_{i}+\partial_{t}\left(-\frac{2}{\epsilon N^{2}} h^{1 / 2} N^{\mid i} \psi_{i}\right) \\
& +\partial_{i} \mathcal{V}^{i} .
\end{aligned}
$$


Variation with respect to the induced metric: As the independend variation we take $\delta h_{i j} \equiv \psi_{i j}$. Then, indices are raised and lowered by $h_{i j}$ and $h^{i j}$ (as e.g. $\left.\psi_{j}^{i} \equiv h^{i k} \psi_{k j}\right)$.

$$
\begin{aligned}
\delta \mathcal{L}= & \mathcal{E}^{i j} \psi_{i j}+\frac{\dot{\epsilon}}{2 \epsilon^{2} N} h^{1 / 2}\left(\left(N K+A_{j}^{j}\right) \psi_{i}^{i}-2 A^{i j} \psi_{i j}+h^{i j} \dot{\psi}_{i j}\right) \\
& +\partial_{t}\left(\frac{1}{\epsilon N} h^{1 / 2}\left(A_{j}^{j} \psi_{i}^{i}+\left(N K^{i j}-2 A^{i j}\right) \psi_{i j}+h^{i j} \dot{\psi}_{i j}\right)\right) \\
& +\partial_{i} \mathcal{W}^{i} .
\end{aligned}
$$

In these formulae the $\mathcal{E}$ 's denote the regular, single-signature Einstein equations (i.e. without $\dot{\epsilon}$-terms). The divergence terms (denoted by $\partial_{i} \mathcal{U}^{i}$ etc.), contain expressions involving $\dot{\epsilon}$, and they belong to the sort of contributions we omit when integrated over.

Here, the formal reason for the appearance of $\dot{\epsilon}$-terms apart from those contained in the (full, i.e. distributional) Einstein equations (cf. equation (1.1)) becomes clear. Normally, one expects the metric determinant to absorb all such additional contributions (this is the step from $\delta R[g]$, which we have not displayed, to $\delta \mathcal{L}$ ). However, the function $\epsilon(t)$ does not appear in $|g|^{1 / 2}=N h^{1 / 2}$ because its absolute value $|\epsilon|$ has been set 1 . Clearly, as long as $\epsilon$ is treated as an arbitrary function in $R[g]$, one expects the appearance of anomalous $\epsilon$-terms in $\delta \mathcal{L}$, hence a "mismatch" between the Lagrangian density and the volume element. If $\epsilon$ is a step function with values \pm 1 , one might expect this "mismatch" to disappear. However, this is not the case! Hence, only part of the $\dot{\epsilon}$-terms in $\delta \mathcal{L}$ are due to the (full) Einstein equations as given by (2.7)-(2.9). This is the reason for the field equations point of view being not necessarily equivalent to the Lagrangian point of view. Many troubles with signature change seem to stem from this fact, and it is unavoidable as long as the volume element in the action is defined as an object by its own, namely $|g|^{1 / 2} d^{n} x$ with $|\epsilon|=1$ inserted.

Here we arrive at the second possibility for interpreting the product $|g|^{1 / 2} R[g]$ that will lead us to the version $S_{3}$ of the Einstein-Hilbert action. As already mentioned above, we formally assume $|g|^{1 / 2}=|\epsilon|^{1 / 2} N h^{1 / 2}$ and define the modified Lagrangian density

$$
\overline{\mathcal{L}}=|\epsilon|^{1 / 2} \mathcal{L} .
$$


Treating $|\epsilon|^{1 / 2}$ as an arbitrary function, we obtain, from (2.5),

$$
\begin{aligned}
\overline{\mathcal{L}} & =|\epsilon|^{1 / 2} N h^{1 / 2} R[h]+\frac{|\epsilon|^{1 / 2}}{\epsilon} N h^{1 / 2}\left(K^{i j} K_{i j}-K^{2}\right)-\mathcal{F} h^{1 / 2} K \\
& +\partial_{t}\left(-2 \frac{|\epsilon|^{1 / 2}}{\epsilon} h^{1 / 2} K\right)+\partial_{i}\left(2 \frac{|\epsilon|^{1 / 2}}{\epsilon} h^{1 / 2} N^{i} K-2|\epsilon|^{1 / 2} h^{1 / 2} N^{\mid i}\right),
\end{aligned}
$$

where

$$
\mathcal{F}=\left(\frac{\partial_{t} \epsilon}{\epsilon}-\frac{\partial_{t}|\epsilon|}{|\epsilon|}\right) \frac{|\epsilon|^{1 / 2}}{\epsilon} .
$$

Hence, the net effect of the transition from $\mathcal{L}$ to $\overline{\mathcal{L}}$ is (apart from the $|\epsilon|^{1 / 2}$-factors) that $\dot{\epsilon} / \epsilon^{2}$ has been replaced by $\mathcal{F}$. Inserting $|\epsilon| \equiv 1$ returns of course $\dot{\epsilon} / \epsilon^{2}$. An alternative interpretation is to "regularize" everything by looking at $\mathcal{F}$ as $\epsilon$ were an arbitrary (say, smooth) function. Then $\mathcal{F}(t)=0$ for all $t$ such that $\epsilon(t) \neq 0$. Whether the zeros of a smooth version of $\epsilon(t)$ produces a non-trivial distribution is a matter of interpretation, because $\mathcal{F}(t)$, as it stands, is not well-defined. Its two contributions produce bad singularities when $\epsilon(t)=0$. Thus a possible interpretational scheme for an alternative version of the Einstein-Hilbert action is to set $\mathcal{F} \equiv 0$ by hand. This is what we will do here, in order to define $\overline{\mathcal{L}}$ unambigously. However, note that this interpretations does not allow $|g|^{1 / 2}$ and $R[g]$ to be objects by their own that are just multiplied. The reason for this alternative Lagrangian density to exist is the freedom in choosing the order in which $\epsilon$ is (a) "regularized" and (b) set equal \pm 1 in the various quantities. Another way of looking at this is to insist on the Lagrangian density $\overline{\mathcal{L}}$ being the ordinary product of $|g|^{1 / 2}$ (as used in the definition of $S_{1}$ ) with "the" Ricci scalar. This amounts to propose a modified definition for the Ricci scalar of a signature changing metric by $\overline{\mathcal{L}}=N h^{1 / 2} \bar{R}[g]$. It would be interesting to study the properties of $\bar{R}[g]$ (in comparison to $R[g]$ ) in more detail than is possible here.

The variations of $\overline{\mathcal{L}}$ proceed along the same lines as those given above. In order to be brief, we just write down the overall structure (obtained without setting $\mathcal{F} \equiv 0$, for the moment)

$$
\delta \overline{\mathcal{L}}=\overline{\mathcal{E}}+\mathcal{F} \mathcal{Z}+\mathcal{G}+\partial_{\mu} \mathcal{X}^{\mu}
$$

where $\overline{\mathcal{E}}$ stands for the single-signature Einstein equations (without $\dot{\epsilon}$-terms), $\mathcal{Z}$ is an expression not containing any $\epsilon$, and

$$
\mathcal{G}=\frac{|\epsilon|^{1 / 2} \partial_{t} \epsilon}{2 \epsilon^{2}} h^{1 / 2} K \psi_{i}^{i}-\frac{\partial_{t}|\epsilon|}{2 \epsilon|\epsilon|^{1 / 2}} h^{1 / 2} K^{i j} \psi_{i j}
$$


If the identification $\mathcal{F} \equiv 0$ is made, this reduces to

$$
\mathcal{G}=\frac{|\epsilon|^{1 / 2} \partial_{t} \epsilon}{2 \epsilon^{2}} \widetilde{\pi}^{i j} \psi_{i j}
$$

In this case, apart from the divergence term, the variation of $\overline{\mathcal{L}}$ fits into the scheme $G_{\mu \nu}[g] \delta g^{\mu \nu}$, with $G_{\mu \nu}$ being the full Einstein tensor (including some $\dot{\epsilon}$-terms, as is easily obtained from the full Ricci tensor (2.7)-(2.9) in a zero shift coordinate system). As a consequence, the "additional terms" in (1.1) vanish (if the off-shell variations are constrained by a differentiability condition, see Section 5), and one recovers the Einstein equations $R_{\mu \nu}=0$ being equivalent to the variational problem defined by the Lagrangian density $\overline{\mathcal{L}}$.

A further alternative, that will not be persued here, is to define the volume element using the complex valued expression $(-g)^{1 / 2}$ instead of $|g|^{1 / 2}$. This is closer to the spirit of the "Wick rotation" in quantum field theory, and to what one does in Euclidean quantum gravity, and it produces an additional $i$ in the Euclidean sector. Although an action generated along these lines may be well suited as an exponent in a path integral, it does not define a well-posed classical variational problem. Its advantage is analyticity rather than reality. Maybe some of the misunderstandings in the topic of signature change are due to the appearance of this (formal) alternative.

When applied to a metric which is in Gaussian form, the expression for $\delta \mathcal{L}$ simplifies considerably. Let us note in particular that all contributions originating from the variation of the shift vector (2.12) vanish except for the standard Einstein equation term $\mathcal{E}^{i}$ and a spatial divergence. This is important, because it suspends us from dealing with non-zero shift metrics. From now on we will ignore variations with respect to $N_{i}$.

Having noticed this simplification, the variational principle is defined as follows: Consider a metric in Gaussian form

$$
d s^{2}=-\epsilon(t) d t^{2}+h_{i j}(t, \mathbf{x}) d x^{i} d x^{j}
$$

and try to solve the equation $\delta S=0$ for variations off this given metric. Part of the information contained in this equation are the single-signature Einstein equations off the hypersurface $\Sigma$. We assume these to be already satisfied (hence $\mathcal{E}=\mathcal{E}^{i}=\mathcal{E}^{i j}=0$ and $\overline{\mathcal{E}}=0$ in the alternative approach to the volume element problem). The remaining information in $\delta S=0$ is only relevant at $\Sigma$, and thus is either inconsistent or leads 
to junction conditions, expressed in terms of Gaussian coordinates. The minimal $\grave{a}$ priori requirement for the functions $h_{i j}$ in (2.20) is that they are continuous in $t$ across $\Sigma$ and constitute a strictly positive matrix. The independent variations to be considered from now on are $\delta N$ and $\delta h_{i j} \equiv \psi_{i j}$. We assume these to be continuous across $\Sigma$ as well. (As will turn out, the variations induced by $\delta N$ to not produce any new information, once the implications of $\delta S / \delta h_{i j}=0$ are exploited). The structure of (2.20) implies that $\delta h_{i j}$ provides a variation within the class of Gaussian form

metrics. As a consequence, $h_{i j}+\delta h_{i j}$ defines a prototype for an off-shell metric and has to be continuous across $\Sigma$ as well. Note that this class of off-shell metrics (within which all variations are performed) - although Gaussian as (2.20) - is conceptually different from the class of solutions (on-shell metrics). So far, we have said that off-shell metrics must have continuous $h_{i j}$. As we will see, most candidate actions require an additional (⿳亠口冋 priori) restriction of the class of off-shell metrics (and thus of off-shell variations $\delta h_{i j}$ ) in order to define a Lagrangian model at all. The restrictions that will appear are:

(i) the $h_{i j}$ are $C^{1}$ across $\Sigma$ (i.e. $\partial_{t} h_{i j}$ exists on $\Sigma$ ), or

(ii) the $h_{i j}$ are $C^{1}$ across $\Sigma$, and $\partial_{t} h_{i j}=0$ there.

To what extent the class (ii) can be phrased "off-shell" is a matter of taste. In all cases admitting solutions to the variational problem at all, these solutions (the on-shell metrics) will (apart from the regular Einstein-equations off $\Sigma$ ) satisfy the condition (i) (which is identical to the junction conditions for weak signature change) or, in addition, (ii) (which is identical to the junction conditions for strong signature change). We have laid some emphasis on these issues because the usual notion of "arbitrary variation" is too rough for signature change.

\section{Candidate actions}

The setting for the action integrals is as follows: Consider a single signature change at $\Sigma \equiv \Sigma_{0}$. Assume $\Sigma$ to be spacelike with respect to its Lorentzian side. Denote the total manifold by $\mathcal{M}$, and its domains $(t<0, t>0)$ by $\left(\mathcal{M}_{-}, \mathcal{M}_{+}\right)$. In the case of global complications, redefine $\mathcal{M}$ to be a sufficiently close neighbourhood of $\Sigma$. Let $\epsilon_{ \pm} \equiv \epsilon\left[g_{ \pm}\right]$denote the metric signature in these domains $\left(\epsilon[g]=\epsilon_{-} \Theta(-t)+\epsilon_{+} \Theta(t)\right.$ being the discontinuous "covariant" signature expression for the total manifold $\mathcal{M}$; its precise value at $\Sigma$ will never be important). In the case of a true signature change we have $\epsilon_{+}=-\epsilon_{-}(=1$ or -1$)$. However, for comparison we keep the possibility $\epsilon_{+}=\epsilon_{-}(=1$ or -1$)$. From now on, sub- or superscripts \pm will distinguish quantities 
due to $\mathcal{M}_{ \pm}$.

The singular actions are (formally) defined as

$$
S_{1}=\int_{\mathcal{M}} d^{n} x \mathcal{L}
$$

and

$$
S_{2}=\int_{\mathcal{M}} d^{n} x \epsilon[g] \mathcal{L}
$$

As explained in the previous Section, $S_{1}$ provides one interpretational version of the Einstein-Hilbert action. The other version is defined by (using $\overline{\mathcal{L}}$ from (2.15) with $\mathcal{F} \equiv 0)$

$$
S_{3}=\int_{\mathcal{M}} d^{n} x \overline{\mathcal{L}}
$$

For completeness, we also introduce the analogue of $S_{2}$ within this alternative scheme

$$
S_{4}=\int_{\mathcal{M}} d^{n} x \epsilon[g] \overline{\mathcal{L}}
$$

We should add here that ony might hope the principle of "general covariance" to single out $S_{1}$ or $S_{3}$ as the "correct" action generalizing the standard $S_{E H}$. At least, in ordinary general relativity, one is of course not free to modify the volume element by will. However, in a theory admitting a change of signature, an additional coordinateindependent object arises, namely the hypersurface $\Sigma$ at which the change occurs, together with its geometric (intrinsic and extrinsic) structur. Accordingly, the difference $S_{3}-S_{1}$ is just a surface integral over $\Sigma$. Inserting $\left|\epsilon_{ \pm}\right|=1$, we get

$$
S_{3}=S_{1}+\left(\epsilon_{+}-\epsilon_{-}\right) \int_{\Sigma} d^{n-1} x h^{1 / 2} K
$$

hence none of the two actions can be excluded by covariance arguments. As we have already stated in Section 2, the variational principle defined by $S_{3}$ is equivalent to the full (distributional) Einstein equations. Hence, equation (3.5) shows that the straightforward generalization of $S_{E H}$, namely $S_{1}$, must be modified by a hypersurface integral in order to reproduce the full Einstein equations from a Lagrangian model.

The first two regular actions result, as announced, from breaking the singular ones into

$$
S_{5}=-\epsilon\left[g_{-}\right] \int_{\mathcal{M}_{-}} d^{n} x\left|g_{-}\right|^{1 / 2} R\left[g_{-}\right]+\epsilon\left[g_{+}\right] \int_{\mathcal{M}_{+}} d^{n} x\left|g_{+}\right|^{1 / 2} R\left[g_{+}\right]
$$


and

$$
S_{6}=\epsilon\left[g_{-}\right] \int_{\mathcal{M}_{-}} d^{n} x\left|g_{-}\right|^{1 / 2} R\left[g_{-}\right]+\epsilon\left[g_{+}\right] \int_{\mathcal{M}_{+}} d^{n} x\left|g_{+}\right|^{1 / 2} R\left[g_{+}\right]
$$

It proves useful to define

$$
S_{ \pm}=\epsilon\left[g_{ \pm}\right] \int_{\mathcal{M}_{ \pm}} d^{n} x\left|g_{ \pm}\right|^{1 / 2} R\left[g_{ \pm}\right]
$$

then $S_{5}=-S_{-}+S_{+}$and $S_{6}=S_{-}+S_{+}$.

The remaining two regular actions are constructed by subtracing the $\partial_{t}$-term from (2.5), hence they consist of integrations over the Lagrangian densities

$$
\mathcal{L}_{ \pm}=\epsilon_{ \pm} N_{ \pm} h_{ \pm}^{1 / 2} R\left[g_{ \pm}\right]+2 \partial_{t}\left(h_{ \pm}^{1 / 2} K_{ \pm}\right)
$$

In view of the sign structure appearing here, a slight redefinition is in place. So far, the extrinsic curvature $K_{i j}$ has been defined with respect to the normal vector $n^{\mu}$ which is (at $\Sigma$ ) outward directed with respect to $\mathcal{M}_{-}$and inward directed with respect to $\mathcal{M}_{+}$. In order to have a notation at hand that relies on the intrinsic properties of a domain alone, we define $\mathcal{K}_{i j}$ to be the extrinsic curvature with respect to the inward directed normal of each domain, and $\mathcal{K}=\mathcal{K}_{i}^{i} \equiv h^{i j} \mathcal{K}_{i j}$. Hence, $\mathcal{K}_{i j}^{ \pm}= \pm K_{i j}^{ \pm}$and (due to the continuity of $\left.h_{i j}\right) \mathcal{K}_{ \pm}= \pm K_{ \pm}$. As a consequence, the integration over $(3.9)$ results into $\mathcal{S}\left(\mathcal{M}_{ \pm}\right)$, where for any single-signature domain $\mathcal{N}$ we have defined

$$
\mathcal{S}(\mathcal{N})=\epsilon[g] \int_{\mathcal{N}} d^{n} x|g|^{1 / 2} R[g]-2 \int_{\partial \mathcal{N}} d^{n-1} x|h|^{1 / 2} \mathcal{K}
$$

This is a natural construction, and it is very close to the action used in the path integral formulations for quantum gravity [26], [29] and quantum cosmology [1], [2]. Note that $\partial \mathcal{M}_{ \pm}=\Sigma$ for both domains, but with a different orientation. This is equivalent to the fact that the $\partial_{t}$-term in (3.9), when integrated over $t$, produces values on $\Sigma$, but with an additional $\mp$ sign. Since we are only interested in the junction conditions at $\Sigma$, we neglect any contribution from other boundaries of $\mathcal{M}_{ \pm}$ than $\Sigma$. The two remaining candidate actions are thus

$$
S_{7}=-\mathcal{S}\left(\mathcal{M}_{-}\right)+\mathcal{S}\left(\mathcal{M}_{+}\right)
$$

and

$$
S_{8}=\mathcal{S}\left(\mathcal{M}_{-}\right)+\mathcal{S}\left(\mathcal{M}_{+}\right)
$$


By looking at (2.5) we see that the $\epsilon$-prefactors of $S_{ \pm}$and $\mathcal{S}\left(\mathcal{M}_{ \pm}\right)$cancel those in front of the kinetic term in $\mathcal{L}$. For $\epsilon_{+}=-\epsilon_{-}=1$, the actions $S_{5}, \ldots S_{8}$ take the form as described in the introduction. However, had we assumed this standard sign choice from the outset, we would not have noticed the signature pattern provided by the various $\epsilon$ 's that makes $\epsilon[g] R[g]$ a particularly nice alternative to $R[g]$. We are now ready to discuss the eight candidate actions. The properties of the singular actions rely on the explicit $\dot{\epsilon}$-contributions appearing in the variational formulae of Section 2 (these terms do not even show up in the regular actions). The properties of the regular actions are based on the partial $t$-derivative terms that are, from the singular action point of view, just divergences that ought to be omitted.

\section{Singular actions $S_{1}$ and $S_{2}$}

The variation $\delta S_{1}$ is obtained by integrating (2.11) and (2.13) over the total manifold. Assuming the metric considered to be of the Gaussian form, inserting the regular part of the Einstein equations $\mathcal{E}=\mathcal{E}^{i j}=0$ and throwing away the boundary terms, we note that a $\dot{\psi}_{i j}$-term has still survived. Integrating by parts (in the sense of distributions), this can be removed, leading to a term proportional to $\ddot{\epsilon} h^{1 / 2} h^{i j} \delta h_{i j} / \epsilon^{2}$. In the case of a true signature change, $\ddot{\epsilon}$ is of the type $\delta^{\prime}(t)$. If arbitrary off-shell variations $\delta h_{i j}$ that are continuous (or $C^{1}$ ) across $\Sigma$ are admitted, an immediate consequence is $h^{i j}=0$, which is unacceptable. Hence, the standard Einstein-Hilbert action $S_{1}$ (when interpreted as the integral over the product of $|g|^{1 / 2}$ with $R[g]$ as two quantities by their own) does not define a sensible variational principle for signature change. One may of course restrict the class of off-shell metrics to $h_{i j}$ being $C^{1}$ and satisfying $\partial_{t} h_{i j}=0$ at $\Sigma$ (condition (ii) in Section 2). As a consequence, $K_{i j}=0$, and only variations with $\dot{\psi}_{i j} \equiv \partial_{t} \delta h_{i j}=0$ are allowed. This gives $\delta S_{1}=0$, and the junction conditions of strong signature change, but the junction conditions have been assumed rather than derived. Also, the notion of off-shell metrics having to satisfy such a strong condition is not satisfactory at all.

The situation is even worse with $S_{2}$. Multiplying (2.11) and (2.13) by $\epsilon$ and integrating by parts, we obtain the relevant contribution

$$
\frac{\dot{\epsilon}}{2 \epsilon} h^{1 / 2}\left(K \delta N+K \psi_{i}^{i}-2 K^{i j} \psi_{i j}-h^{i j} \dot{\psi}_{i j}\right)
$$


to be integrated over. Here $\dot{\epsilon} / \epsilon$ is not even a well-defined distribution (it is of the type $\operatorname{sgn}(t) \delta(t))$. The only possibility to make the parenthesis vanish is to restrict the class of off-shell metrics to satisfy the strong junction conditions (condition (ii) of Section 2) as above, which is against the spirit of a Lagrangian formalism as well. Alternatively, one may imagine $\epsilon(t)$ to arise as a limit of antisymmetric functions $\epsilon_{\text {smooth }}(t)$. Then formally, $\dot{\epsilon} / \epsilon$ is zero when multiplied by continuous functions. As a consequence, the $\dot{\psi}_{i j}$-term drops out (its continuity may be assumed), and the rest in the parenthesis of (4.1) is continuous if $K_{i j}$ is (which implies weak signature change). This procedure suffers from the ignorance of $|g|^{1 / 2} \equiv N h^{1 / 2}$ against the contributions of $\epsilon_{\text {smooth }}(t)$ before the limit is taken. However, it may be considered as an ad hoc regularization scheme for $S_{2}$.

Thus, the perspectives for using $S_{1}$ and $S_{2}$ as actions are somewhat unpleasant and limited.

\section{$5 \quad$ Singular actions $S_{3}$ and $S_{4}$}

As already stated in Section 2, the variational principle $\delta S_{3}=0$ generates the full Einstein equations. This is the Lagrangian version of the usual point of view that assumes the Einstein equations as first principles. The variational formula (2.17) with $\mathcal{F} \equiv 0$ and (2.19) immediately implies that, upon imposing $\partial_{t} h_{i j}$ to be continuous for the class of off-shell metrics (in order to make $S_{3}$ well-defined; this is the condition (i) of Section 2), the solutions must satisfy $\tilde{\pi}^{i j}=0$ at $\Sigma$. For $n \neq 2$ this is equivalent to $K_{i j}=0$, hence strong signature change. Thus, $S_{3}$ defines a Lagrangian model, as long as one accepts the differentiability condition for the off-shell metrics.

The candidate action $S_{4}$ provides similar problems as $S_{2}$. Instead of expressions like $\dot{\epsilon}|\epsilon|^{1 / 2} / \epsilon^{2}$ (which appear in $\delta S_{3}$ and can - after setting $|\epsilon|=\epsilon^{2}=1$ in the end - be interpreted as ordinary $\delta$-distributions), we are now faced with $\dot{\epsilon}|\epsilon|^{1 / 2} / \epsilon$, which is much less well-defined. The possible (although not very appealing) procedures one may perform are analogous to those mentioned in the discussion of $S_{2}$ in the previous Section. Hence, we skip all details and just summarize that $S_{4}$ is ruled out along the same lines as $S_{2}$. 


\section{$6 \quad$ Regular actions $S_{5}$ and $S_{6}$}

We write down the variations of $S_{ \pm}$off a metric in Gaussian form. Using (2.11) and (2.13), we get

$$
\delta S_{ \pm}=\mp \int_{\Sigma} d^{n-1} x h^{1 / 2}\left(2 K^{ \pm} \delta N+K_{ \pm}^{i j} \psi_{i j}+h^{i j} \dot{\psi}_{i j}^{ \pm}\right) .
$$

Note that the $\epsilon$ 's have cancelled due to the prefactors. Considering now the candidate action $S_{5}=-S_{-}+S_{+}$, we encounter the contribution $h^{i j}\left(\dot{\psi}_{i j}^{+}+\dot{\psi}_{i j}^{-}\right)$which has to vanish in order to allow for solutions of $\delta S_{5}=0$. Again, a restriction of the class of off-shell metrics is necessary. The only natural way to do this is to impose the strong junction conditions (condition (ii) from Section 2) in order to enforce $\dot{\psi}_{i j}^{ \pm}=0$. Thus, $S_{5}$ does not define a sensible model, along with $S_{1}, S_{2}$ and $S_{4}$.

The situation first changes with $S_{6}=S_{-}+S_{+}$. The unpleasant $\dot{\psi}_{i j}^{ \pm}$-terms appear with a relative minus sign, and we are left with

$$
\delta S_{6}=\int_{\Sigma} d^{n-1} x h^{1 / 2}\left(2\left(K^{-}-K^{+}\right) \delta N+\left(K_{-}^{i j}-K_{+}^{i j}\right) \psi_{i j}+h^{i j}\left(\dot{\psi}_{i j}^{-}-\dot{\psi}_{i j}^{+}\right)\right) .
$$

Restricting the class of off-shell metrics to those for which $h_{i j}$ is $C^{1}$ across $\Sigma$ (condition (i) of Section 2), we get $\dot{\psi}_{i j}^{-}-\dot{\psi}_{i j}^{+}=0$. The remaining variational problem leads to $K_{-}^{i j}=K_{+}^{i j}$, hence weak signature change. We conclude that $S_{6}$ defines a sensible model, once the differentiability condition defining the class of off-shell metrics is accepted.

Note that all derivations given in this Section apply for the single-signature case $\epsilon_{+}=\epsilon_{-}$as well. For $S_{6}$ this becomes what we have called the "additivity property" in the introduction: one may (assuming the off-shell- $h_{i j}$ to be $C^{1}$ across $\Sigma$ ) derive the standard Einstein equations by breaking the Einstein-Hilbert action into a sum of two integrals. This may be understood as a naturality argument in favour of $\epsilon[g] R[g]$.

\section{Regular actions $S_{7}$ and $S_{8}$}

These actions provide the least complications, since the "time"-derivative part of (2.5) is missing. One easily finds

$$
\delta \mathcal{S}\left(\mathcal{M}_{ \pm}\right)=\mp \int_{\Sigma} d^{n-1} x h^{1 / 2}\left(K_{ \pm} h^{i j}-K_{ \pm}^{i j}\right) \psi_{i j} \equiv \mp \int_{\Sigma} d^{n-1} x \widetilde{\pi}_{ \pm}^{i j} \psi_{i j}
$$


For $S_{7}=-\mathcal{S}\left(\mathcal{M}_{-}\right)+\mathcal{S}\left(\mathcal{M}_{+}\right)$, this gives an integrand $\left(\widetilde{\pi}_{+}^{i j}+\widetilde{\pi}_{-}^{i j}\right) \psi_{i j}$. Hence, when the off-shell metrics are restricted to continuous $\partial_{t} h_{i j}$ (condition (i) from Section 2 ), we have $\tilde{\pi}_{+}^{i j}=\tilde{\pi}_{-}^{i j}$. As a consequence, we find $\widetilde{\pi}^{i j}=0$ at $\Sigma$ as the solution of the variational problem. For $n \neq 2$ this is equivalent to $K_{i j}=0$ at $\Sigma$. Hence, $S_{7}$ provides a Lagrangian model allowing for strong signature change, once the class of off-shell metrics is characterized by the above differentiability condition.

However, there is another possibility to let $S_{7}$ define a variational problem: If one is willing to give up the continuity of $K_{i j}$ across $\Sigma$ (and hence keeps the class of admissable off-shell metrics unconstrained) the action $S_{7}$ leads to $K_{i j}^{-}+K_{i j}^{+}=0$. This predicts a kink in the induced metric across $\Sigma$ and could in principle be considered as a further (super-weak or "anticontinuous") junction condition by its own [30].

The variation of the last action $S_{8}=\mathcal{S}\left(\mathcal{M}_{-}\right)+\mathcal{S}\left(\mathcal{M}_{+}\right)$leads to the integrand $\left(\widetilde{\pi}_{+}^{i j}-\widetilde{\pi}_{-}^{i j}\right) \psi_{i j}$. Hence, without restricting the class of off-shell metrics (apart from $h_{i j}$ being continuous), this immediately leads to the solution $\widetilde{\pi}_{+}^{i j}=\widetilde{\pi}_{-}^{i j}$ of the variational problem. If $n \neq 2$, this is equivalent to $K_{i j}^{+}=K_{i j}^{-}$. In this way, $S_{8}$ defines a perfectly well-posed variational principle allowing for weak signature change. It is the only candidate action that requires no differentiability assumption for the class of off-shell metrics (but rather predicts $\partial_{t} h_{i j}$ to be continuous). It is thus closer to the spirit of a Lagrangian formalism than all other candidates. Moreover, for $\epsilon_{+}=\epsilon_{-}$, it shows the "additivity property" (and thus leads to the single-signature Einstein equations straightforwardly). In this sense, weak signature change seems favoured from the Lagrangian point of view.

\section{Variations including $\delta \Sigma$}

So far we have fixed the hypersurface $\Sigma$ of signature change. However, a full action principle requires the structure $\left(\mathcal{M}_{-}, g_{-}, \mathcal{M}_{+}, g_{+}\right)$to be interpreted as a single configuration with respect to which the action is varied.

All of our candidate actions may be written as a sum of a volume-integral over $\mathcal{M}_{ \pm}$and a surface-integral over $\Sigma$. Start from an on-shell metric (i.e. satisfying the field equations and junction conditions) and allow $\Sigma$ to be deformed infinitesimally into $\Sigma^{\text {new }}$. Choose an (off-shell) metric that changes sign at $\Sigma^{\text {new }}$. The variation of the volume-integral part typically yields - apart from contributions that vanish on 
account of the field equations - expressions of the form

$$
\left(\int_{\mathcal{M}_{+}^{\text {new }}}-\int_{\mathcal{M}_{+}}\right) d^{n} x \mathcal{L}_{+}
$$

and a similar term for $\mathcal{M}_{-}$. Next we use the fact that the volume part of the Lagrangian densities vanishes at the hypersurface of signature change (see equation (9.3) below for a justification - this carries over to the more general case when matter fields are included: the volume part of the Lagrangian density will not necessarily vanish on-shell in general, but it does so at the hypersurfaces of signature change). Hence we may, to first order, insert $\mathcal{L}_{+}=0$ and find zero variation due to the volumeintegral contributions. The contributions to $\delta S$ that represent surface-integrals turn out to vanish partially due to fact that the junction conditions are satisfied by the old metric at the old hypersurface. However, for those Lagrangians which contain true surface contributions (the integrand being a multiple of $K$ in each case) some terms of the type

$$
\left(\int_{\Sigma_{\text {new }}}-\int_{\Sigma}\right) d^{n-1} x h^{1 / 2} K
$$

remain. In the case of $S_{7}$ and $S_{8}$, these are manifestly absent (as is the case for $S_{3}$ and - in one of the two regularization schemes - for $S_{4}$ ). Thus we find $\delta^{\text {tot }} S_{3,4,7,8}=0$. (In the next section we will see that $S_{7}$ and $S_{8}$ are essentially equivalent to $S_{3}$ and $S_{4}$ ). In the other cases, the condition $\delta S=0$ puts further constraints on the class of admissable off-shell metrics or, if one is not willing to implement these, restricts the set of solutions even further. (To be a bit more specific we note that in the vaccum case the variation might still vanish in some cases because the field equations imply $\partial_{t} K_{ \pm}=0$ at $\Sigma$, but when a cosmological constant is included, this generalizes to $\partial_{t} K_{ \pm}=-2 \epsilon_{ \pm} N \Lambda /(n-2)$, where $\left.n=\delta_{\mu}^{\mu}\right)$.

Summarizing, we found that for the most interesting actions $S_{3,4,7,8}$ the variation of the signature change hypersurface $\Sigma$ does not provide any new information.

\section{$9 \quad S_{7}$ and $S_{8}$ revisited}

Putting our previous results together, the two candidate actions $S_{7}$ and $S_{8}$ provide the most reasonable actions for weak and strong signature change, respectively. (We leave apart the possibility of obtaining an "anticontinuous" junction condition from $S_{7}$, as was mentioned in Section 7). So far, these action functionals have been 
defined in a "covariant" (i.e. coordinate-independent) way (see (3.11) and (3.12)). However, it is instructive to write down the expressions for $S_{7}$ and $S_{8}$ in terms of a coordinate system in which the metric is given by (2.1), with $N, N_{i}$ and $h_{i j}$ being continuous and $\Sigma$ described by the equation $t=0$. The extrinsic curvature $K_{i j}$, as given by (2.2), plays (apart from the lapse factor and the shift contribution) the role of a "velocity", with $h_{i j}$ being the basic dynamical variable.

Joining the two integrals of (3.11) into a single one, we may write

$$
S_{7}=\int_{\mathcal{M}} d^{n} x N h^{1 / 2}\left(\widetilde{\epsilon}_{1}\left(K^{i j} K_{i j}-K^{2}\right)+\widetilde{\epsilon}_{2} R[h]\right)
$$

where, $\widetilde{\epsilon}_{1}(t)=\operatorname{sgn}(t)$ and $\tilde{\epsilon}_{2}(t)=-\epsilon_{-} \Theta(-t)+\epsilon_{+} \Theta(t)$. If $\epsilon_{+}$and $\epsilon_{-}$are negative to each other, $\widetilde{\epsilon}_{2}(t)$ is constant. In particular, for $\epsilon_{+}=-\epsilon_{-}=1$, we get $\widetilde{\epsilon}_{2}(t)=1$. As a by-product we note that, applying (3.3) for (2.15) with $\mathcal{F} \equiv 0$, and inserting $|\epsilon|=1$ (which is allowed in this place, due to our construction), we find the same expression, hence $S_{3}= \pm S_{7}$ if $\epsilon_{+}=-\epsilon_{-}=1$. The non-standard definition of $|g|^{1 / 2} R[g]$ just generates the same action as the two-domain construction for $S_{7}$. Martin also arrives at an identical expression (in a slightly more heuristic way) [14]. Note that $K_{i j}$ contains a factor $1 / N$, so that (when expressed in terms of the "velocities" $\left.\dot{h}_{i j}\right)$, the overall structure of this expression is " $(1 / \mathrm{N})$ kinetic energy N potential energy". The variation with respect to $N$ generates the Hamiltonian constraint equation (although in Lagrangian form, see equation (9.3) below). The variation with respect to $h_{i j}$ (generating the time evolution equations) produces the $\delta$-function terms contained in the full Einstein equations, hence the junction conditions for strong signature change.

One may bring $S_{8}$ into an analogous form by joining the two integrals of (3.12) into a single one. The reason for this is that the construction of $S_{8}$ is just the covariant formulation of the recipe to omit the $\partial_{t}$ and $\dot{\epsilon}$-terms in $\epsilon \mathcal{L}$, with $\mathcal{L}$ given by (2.5). Hence,

$$
S_{8}=\int_{\mathcal{M}} d^{n} x N h^{1 / 2}\left(K^{i j} K_{i j}-K^{2}+\epsilon R[h]\right) .
$$

The factor $\epsilon$ arises here straightforwardly. (Let us note in parentheses that the regularization scheme for $S_{4}$ that puts $\dot{\epsilon}|\epsilon|^{1 / 2} / \epsilon$ effectively equal to zero yields the same expression, hence in this sense $S_{4}=S_{8}$. A similar argument shows that, in the same sense, $\left.S_{2}=S_{6}\right)$. Again, the overall structure is " $(1 / \mathrm{N})$ kinetic energy - N potential energy", but with the signs (and sign changing terms) distributed 
differently as compared to (9.1). In contrast to $S_{7}$, the variation with respect to $h_{i j}$ does not produce any $\delta$-functions. The field equations just contain $\epsilon$ in a purely algebraic way, together with $\dot{K}^{i j}$ as "accelerations", which are thus predicted to undergo a finite jump. Hence, upon integration, the "velocitiy"-type quantities $K_{i j}$ must be well-defined across $\Sigma$, which is identical to the junction conditions for weak signature change. Note also that the sign structure appearing in these two actions corresponds to that of the toy model Lagrangians (1.2) and (1.3) given in the introduction.

The constraint equation, $\delta S / \delta N=0$, is the same for both actions if $\epsilon_{+}=-\epsilon_{-}=$ 1 , and reads

$$
K^{i j} K_{i j}-K^{2}=\epsilon R[h] .
$$

Thus, whenever $\epsilon$ changes sign, the continuity of the extrinsic curvature (which is a consquence in both models) implies that at signature change all solutions must satisfy

$$
K^{i j} K_{i j}-K^{2}=R[h]=0 .
$$

In the model $S_{7}$, the additional condition is $K_{i j}=0$. In any case, the Lagrangian density vanishes at $\Sigma$ (this was used in a somewhat different picture in the previous Section when $\delta \Sigma$ was carried out).

In a Friedmann-Robertson-Walker model with $N=1$ and scale factor $a(t)$, the kinetic terms are given by $K^{i j} K_{i j}-K^{2}=-(n-1)(n-2) \dot{a}^{2} / a^{2}$, which is non-positive (contrary to the generic case). As a consequence, (9.4) implies $\dot{a}=0$, hence $K_{i j}=0$. Thus, in this highly symmetric case, weak signature change automatically implies the strong junction conditions. This remains true if a cosmological constant is included, but already fails for the case of an additional scalar field, although Hayward [23] seems to claim the contrary in his criticism of Ellis et al [6]: his equations (23) - version December 1994 - do admit true weak signature changing solutions in the sense we use this notion. In terms of $t$ they have discontinuous but bounded $\partial_{t t} a$, but when expressed in terms of a "continuous" approach time coordinate $\tau$ (cf. equation 1.5), these solutions display singular second derivatives, $\partial_{\tau \tau} a \sim \epsilon\left|\tau-\tau_{0}\right|^{-1 / 2}$, which is the reason for Hayward not to talk about them at all. This is an example of different results based on different first principles.

We would like to add a remark concerning a possible restriction of the signature change models. This requires to add a cosmological constant contribution to all actions. The inclusion of a cosmological constant is achieved by replacing

$$
R[g] \rightarrow R[g]-2 \Lambda, \quad R[h] \rightarrow R[h]-2 \Lambda .
$$


The proposal we have in mind starts from the fact that, so far, we are not able to be predict whether a signature change will actually occur when it is possible (e.g. when $R[h]$ becomes zero in the $S_{8}$-model; cf. the remarks made in the introduction). This is reflected by the fact that the signature dependent factors $\epsilon$ and $\widetilde{\epsilon}_{2}$ in the expressions (9.1) and (9.2) above are not functionals of the dynamical variables $h_{i j}$. Their dynamical status is actually rather obscure in a Lagrangian (or Hamiltonian) formalism, and when trying to quantize these models, one wonders how to treat them (cf. Ref. [14]). Classically, from (9.3), one reads off that $\epsilon$ is the sign of $\left(K^{i j} K_{i j}-\right.$ $\left.K^{2}\right) R[h]$, and inserting this into $S_{8}$ reveals its involved structure. Moreover, the coordinate system used here restricts any possible signature change to occur at a hypersurface of constant $t$. Another coordinate system would produce signature changes along different hypersurfaces. However, inspired by quantum cosmology, one may think about fixing a cosmologically preferred coordinate system (e.g. by requiring the shift vector $N_{i}$ to vanish and $N$ to depend only on $t$ ). Such a choice is dynamically consistent within the framework of minisuperspace models, where part of the degrees of freedom are frozen and integrated over. Let us denote the "potential" occuring in such models by $2 \Lambda-R[h]$, although $R[h]$ might actually be an integrated version of the Ricci scalar. When the universe "is born" at small geometries (which implies $2 \Lambda-R[h]<0$ ) with Euclidean signature $(\epsilon=-1$ ), it will eventually "evolve" (with respect to $t$ ) towards the conditions for a signature change. An ad hoc assumption resolving the non-uniqueness problem for solutions is to postulate that whenever $2 \Lambda-R[h]<0$ (which is often called the Euclidean sector in superspace) the signature is Euclidean $(\epsilon=-1)$, and whenever $2 \Lambda-R[h]>0$, the signature is Lorentzian $(\epsilon=1)$. This is (together with the initial condition) just the statement that a signature change will occur whenever it is possible. Upon inserting $\epsilon=\operatorname{sgn}(2 \Lambda-R[h])$ into $S_{8}, \epsilon$ has become a functional of the dynamical variables. This modification gives a nice expression, namely

$$
S_{8}^{\prime}=\int_{\mathcal{M}} d^{n} x N h^{1 / 2}\left(K^{i j} K_{i j}-K^{2}-|2 \Lambda-R[h]|\right) .
$$

A ten-dimensional version of this action within the framework of a FriedmannRobertson-Walker (FRW) minisuperspace model may be found in Ref. [16] (where this type of signature change is shown to stabilize internal dimensions). Here, quantization is straightforward (at least formally), and one obtains a Wheeler-DeWitt equation [28] with the "potential term" being replaced by its alsolute value. Similar constructions are of course possible when matter fields are included in addition (or instead of) the cosmological constant. The modification of $S_{7}$ along the same lines 
gives a model that is a bit more involved, and (classically) allows for less signature changes than $S_{8}$. The quantization of such a model seems more problematic (naively, one would obtain the same Wheeler-DeWitt equation as for $S_{8}^{\prime}$ ), but being quantized does not seem to be the intention of strong signature change anyway (as a classical model of quantum tunneling).

Finally, we give the overall structure of $S_{7}, S_{8}$ and $S_{8}^{\prime}$ in the simplest case of a four-dimensional FRW model with cosmological constant by writing down the Lagrangians

$$
\begin{aligned}
\mathcal{L}_{7}^{\mathrm{FRW}} & =-\operatorname{sgn}(t) \dot{a}^{2} / N+N\left(a^{2}-\Lambda a^{4}\right), \\
\mathcal{L}_{8}^{\mathrm{FRW}} & =-\dot{a}^{2} / N+N \operatorname{sgn}(t)\left(a^{2}-\Lambda a^{4}\right), \\
\mathcal{L}_{8}^{\mathrm{FRW}} & =-\dot{a}^{2} / N-N\left|a^{2}-\Lambda a^{4}\right|,
\end{aligned}
$$

where the scale factor and the lapse function have been conveniently redefined.

\section{Conclusion}

Our analysis has singled out $S_{3}, S_{4}$ (in a rather subtle interpretation), $S_{6}, S_{7}$ and $S_{8}$ to define more or less acceptable models for signature change $\left(S_{3}\right.$ and $S_{7}$ for the strong, the others for the weak junction conditions) in any total space-" time" dimension $n \geq 3$. Moreover we found that $S_{3}$ is essentially identical to $S_{7}$ (and in some sense $S_{4}$ to $S_{8}$ ). Straightforward quantization might be conceptually problematic for $S_{6}$, because of the off-shell metric differentiability conditions, the variation of the hypersurface $\Sigma$ (Section 8) being problematic as well. Hence, one should consider $S_{7}\left(S_{8}\right)$ as the best model for strong (weak) signature change, fitting into the Lagrangian framework as regular actions $\left(S_{7}\right.$, when defined as $S_{3}$, as a singular action as well, and $S_{8}$, when defined as $S_{4}$, as a singular action in a particular regularization scheme). None of these two models can à priorily be excluded. This establishes both versions of signature change as Lagrangian models. The regular actions seem to be better suited for a variational description of signature change than the singular ones. In addition we observed that $S_{7}$ needs a differentiability condition for the class of off-shell metrics, whereas $S_{8}$ defines a perfectly well-defined variational problem if just a simple continuity condition is imposed. In this sence, the Lagrangian point of view slightly favours weak over strong signature change. (In additon, as was mentioned in Section 7, the relaxation of the conditions on the class of off-shell metrics 
for $S_{7}$ leads to an "anti-continuous" junction condition as a third version of signature change. It predicts a kink in $h_{i j}$, hence a non-differentiable induced metric, but cannot be excluded either (⿳亠口冋 priorily) within the regular actions approach).

The way we proceeded is something like an "inverse philosophy" as compared to the usual approaches. The models associated with strong signature change require more severe restrictions on the off-shell metrics than the weak models in order to get things well-defined. Normally, one would interpret these restrictions (in particular $K_{i j}=0$ ) as an argument in favour of strong signature change. However, in a Lagrangian framework one is interested in a large class of off-shell metrics - which favours $S_{8}$ against $S_{7}$. This seems to correspond with the different aims pursued with the two models. Strong signature change, when envisaged as a genuinely classical model, might not need a Lagrangian formulation, whereas weak signature change might be quantized, the goal being an alternative to standard quantum cosmology.

The full power of $S_{8}$ becomes visible if it is rephrased as follows: Divide $\mathcal{M}$ into a finite (or sufficiently nice discrete) set of domains $\mathcal{M}_{J}$ inside which the metric has constant signature (and such that all boundaries $\partial \mathcal{M}_{J}$ are spacelike whenever this is well-defined). Moreover, let the corresponding induced metrics at the boundaries be continuous. Define the action to be

$$
S=\sum_{J} \mathcal{S}\left(\mathcal{M}_{J}\right)
$$

Assuming local infinitesimal variations of this situation, the condition $\delta S=0$ produces weak signature change across those boundaries $\partial \mathcal{M}_{J}$ at which $\epsilon[g]$ is discontinuous, and the standard (Lorentzian or Euclidean) Einstein equations everywhere else. Thus, in this framework, weak signature change appears at an equal footing with the "additivity property" of the Einstein-Hilbert action (with boundary term removed), and hence seems to be a natural generalization thereof. This is in turn linked with the conceptual advantages of the regular actions as compared to the singular ones. Moreover, these issues seem to relate to the original motivation for subtracting a boundary term from the Einstein-Hilbert action in quantum gravity [26]

Can one draw a simple conclusion from all these details? In my opinion it is the following: Writing down the integrand of the Einstein-Hilbert action, one encounters distributional and even worse terms (i.e. terms involving $\dot{\epsilon}$ in our language). Among all attempts we tested, exactly those who effectively remove these terms led to an acceptable variational principle. As a consequence, one could think about giving up 
all "distributional" interpretations of what happens at $\Sigma$, and to adopt the idea of regular actions, which are perfectly well-defined within each domain. Once this is done, it appears as a viable alternative to reverse the sign in the Euclidean domains (which, in addition, improves the well-posedness of the action and the properties of the admissible off-shell variations). In this way, both strong and weak signature change emerge as Lagrangian models.

Summarizing, let us state that the Lagrangian approach clearly reveals the existence of (essentially two) different generalizations of general relativity allowing for a classical change of metric signature, none of them being à priori the "correct" one. This justifies our convention to talk about weak and strong signature change as two phenomena, appearing within different models, which are based on different first principles. Further pros and cons are of course still possible and can be based on arguments that concern physical predictions, mathematical or physical richness, naturality or even aestetics.

\section{Note added}

Due to discussions that took place after the original manuscript was prepared, I would like to add a comment on the singular actions aproach.

As was stated in Section 2, the spacial object $K_{i j}$ coincides with the (invariantly defined) extrinsic curvarure only if $|\epsilon|=1$. However, in part of the derivations we have treated $\epsilon$ as if it were an arbitrary function (and sometimes called this procedure a "regularization") - with the exception that $K_{i j}$ is always kept as a quantity without further reference to $\epsilon$. One may however replace at any stage of the "regularization" procedures $K_{i j}=|\epsilon|^{1 / 2} K_{i j}^{\text {true }}$ and keep $K_{i j}^{\text {true }}$ as variable without further reference to $\epsilon$. Doing so in the derivation of an expression for the Ricci scalar $R[g]$, reshuffling partial $t$-derivatives, and performing a similar "regularization" as $\mathcal{F}=0$, we obtain precisely the expression for $R[g]$ as computed within a manifestly "continuous" language, using the "time"-coordinate $\tau$ (cf. the appendix of Ref. [18]), i.e. without $\delta$-function terms. Proceeding with this definition of the Ricci-scalar, and setting $g^{1 / 2}=|\epsilon|^{1 / 2} N h^{1 / 2}$, one would end up again with $\overline{\mathcal{L}}$, hence the action $S_{3}$. This is another example of the fact that the order in which one "regularizes" and sets $|\epsilon|=1$ may not be changed without changing the resulting quantities as well. It illustrates that not even the Ricci-scalar $R[g]$ is unambigously well-defined if $K_{i j} \neq 0$.

In a manifestly "continuous" language, these difficulties re-appear whenever 
terms like $|\tau|^{1 / 2}$ have to be differentiated and thereafter multiplied by singular objects (like $1 / \tau)$. Formally using the product rule for derivatives, one may generate additional $\delta$-functions easily. Also, the rule $\partial_{\tau}(1 / \tau)=-1 / \tau^{2}$ is problematic in the sense of distributions if one likes to look at $1 / t^{2}$ as being the square of $1 / t$. Hence, reshuffling derivatives in the standard formulae for the curvature quantities (which produces different but equivalent versions of one equation in the usual case) may give rise to truly different results when $g_{\tau \tau}(\tau)$ has a zero. All these difficulties show that the regular actions approach is better suited for signature change.

\section{Acknowledgments}

I would like to acknowledge a few very good discussions with Marcus Kriele on the pros and cons. Moreover, he detected an error in the original manuscript. Thanks also to Tevian Dray for pointing me out that one may in principle admit a third version of signature change (see Ref. [30]).

\section{References}

[1] J. B. Hartle and S. W. Hawking, "Wave function of the universe", Phys. Rev. D 28, 2960 (1983).

[2] S. W. Hawking, "The quantum state of the universe", Nucl. Phys. B 239, 257 (1984).

[3] G. W. Gibbons and J. B. Hartle, "Real tunneling geometries and the large-scale topology of the universe", Phys. Rev. D 42, 2458 (1990).

[4] S. A. Hayward, "Signature change in general relativity", Class. Quantum Grav. 9, 1851 (1992).

[5] S. A. Hayward, "On cosmological isotropy, quantum cosmology and the Weyl curvature hypothesis", Class. Quantum Grav. 10, L7 (1993).

[6] G. Ellis, A. Sumeruk, D. Coule and C. Hellaby, "Change of signature in classical relativity", Class. Quantum Grav. 9, 1535 (1992).

[7] G. F. R. Ellis, "Covariant Change of Signature in Classical Relativity", Gen. Relativ. Gravit. 24, 1047 (1992). 
[8] T. Dereli and R. W. Tucker, "Signature dynamics in general relativity", Class. Quantum Grav. 10, 365 (1993).

[9] R. Kerner and J. Martin, "Change of signature and topology in a fivedimensional cosmological model", Class. Quantum Grav. 10, 2111 (1993).

[10] T. Dray, C. A. Manogue and R. W. Tucker, "Particle Production from Signature Change", Gen. Relativ. Gravit. 23, 967 (1991).

[11] T. Dray, C. A. Manogue and R. W. Tucker, "Scalar field equation in the presence of signature change", Phys. Rev. D 48, 2587 (1993).

[12] C. Hellaby and T. Dray, "Failure of standard conservation laws at a classical change of signature", Phys. Rev. D 49, 5096 (1994).

[13] T. Dray and C. Hellaby, "The patchwork divergence theorem", to appear in $J$. Math. Phys, preprint gr-qc/9404002.

[14] J. Martin, "Hamiltonian quantization of general relativity with the change of signature", Phys. Rev. D 49, 5086 (1994).

[15] F. Embacher, "Comments on signature change and the multi-dimensional Wheeler-DeWitt equation", Talk given at the International School-Seminar "Multidimensional Gravity and Cosmology", Yaroslavl, June 1994, to appear in the proceedings, Gravitation and Cosmology 1 (1995), also preprint grqc/9409016.

[16] F. Embacher, "Signature change induces compactification", University Vienna preprint UWThPh-1994-47, also preprint gr-qc/9410012.

[17] F. Embacher, "The trace left by signature-change-induced compactification", University Vienna preprint UWThPh-1994-55, also preprint gr-qc/9411028.

[18] M. Kossowski and M. Kriele, "Smooth and discontinuous signature type change in general relativity", Class. Quantum Grav. 10, 2363 (1993).

[19] M. Kossowski and M. Kriele, "Signature type change and absolute time in general relativity", Class. Quantum Grav. 10, 1157 (1993). 
[20] M. Kossowski and M. Kriele, "Transverse, type changing, pseudo riemannian metrics and the extendability of geodesics", Proc. R. Soc. Lond. A 444, 297 (1994).

[21] M. Kossowski and M. Kriele, "The Einstein equation for signature type changing spacetimes", Proc. R. Soc. Lond. A 446, 115 (1994).

[22] M. Kriele and J. Martin, "Black holes, cosmological singularities and change of signature", preprint gr-qc/9411063.

[23] S. A. Hayward, "Junction conditions for signature change", preprint grqc/9303034.

[24] M. Carfora and G. Ellis, "The geometry of classical change of signature", preprint $\mathrm{gr-q \textrm {c } / 9 4 0 6 0 4 3 .}$

[25] C. W. Misner, K. S. Thorne and J. A. Wheeler, Gravitation, Freeman (San Francisco, 1973).

[26] G. W. Gibbons and S. W. Hawking, "Action integrals and partition functions in quantum gravity", Phys. Rev. D 15, 2752 (1977).

[27] M. B. Green, J. H. Schwarz and E. Witten, Superstring Theory, Vols. 1,2, Cambridge University Press (Cambridge, 1987).

[28] B. DeWitt, "Quantum Theory of Gravity. I. The Canonical Theory", Phys. Rev. 160, 1113 (1967).

[29] S. W. Hawking, "The path-integral approach to quantum gravity", in: S. W. Hawking and W. Israel (eds.), General relativity: An Einstein Centenary Survey, Cambridge University Press (Cambridge, 1979).

[30] I would like to thank T. Dray for pointing this out to me. The possibility of "anticontinuous" junction conditions is briefly mentioned in Ref. [12], analogous junction conditions for a scalar field are considered in Refs. [10], [11], and [31] (see also Ref. [32]).

[31] T. Dray, C. A. Manogue and R. W. Tucker, "Boundary conditions for the scalar field in the presence of signature change", preprint Er-qc/9501034. 
[32] S. A. Hayward, "Comment on 'Boundary conditions for the scalar field in the presence of signature change' ", preprint gr-qc/9502001. 